\section{(6) OPEN ACCESS}

\title{
Proarrhythmic remodelling of the right ventricle in a porcine model of repaired tetralogy of Fallot
}

\author{
David Benoist, ${ }^{1,2}$ Virginie Dubes, ${ }^{1,2}$ François Roubertie, ${ }^{1,2,3}$ Stephen H Gilbert, ${ }^{1,2,4}$ \\ Sabine Charron, ${ }^{1,2}$ Marion Constantin ${ }^{1,2^{2}}$ Delphine Elbes, ${ }^{1,2,5}$ Delphine Vieillot, ${ }^{6}$ \\ Bruno Quesson, ${ }^{1,2}$ Hubert Cochet, ${ }^{1,2,3}$ Michel Haïssaguerre, ${ }^{1,2,3}$ Caroline Rooryck, $^{7}$ \\ Pierre Bordachar, ${ }^{1,2,3}$ Jean-Benoit Thambo, ${ }^{1,2,3}$ Olivier Bernus ${ }^{1,2}$
}

\begin{abstract}
- Additional material is published online only. To view please visit the journal online (http://dx.doi.org/10.1136/ heartjnl-2016-309730)
\end{abstract}

For numbered affiliations see end of article.

\section{Correspondence to} Dr David Benoist, IHU LIRYC, PTIB-Hôpital Xavier Arnozan, Avenue Haut Leveque, Pessac 33604, France; david benoist@u-bordeaux.fr

DB and VD contributed equally.

Received 1 April 2016 Revised 27 June 2016 Accepted 21 July 2016 Published Online First 10 October 2016

\section{CrossMark}

To cite: Benoist D, Dubes $\checkmark$, Roubertie F, et al. Heart 2017:103:347-354.

\section{ABSTRACT}

Objective The growing adult population with surgically corrected tetralogy of Fallot (TOF) is at risk of arrhythmias and sudden cardiac death. We sought to investigate the contribution of right ventricular (RV) structural and electrophysiological remodelling to arrhythmia generation in a preclinical animal model of repaired TOF (rTOF).

Methods and results Pigs mimicking rTOF underwent cardiac MRI functional characterisation and presented with pulmonary regurgitation, RV hypertrophy, dilatation and dysfunction compared with Sham-operated animals (Sham). Optical mapping of rTOF RV-perfused wedges revealed a significant prolongation of RV activation time with slower conduction velocities and regions of conduction slowing well beyond the surgical scar. A reduced protein expression and lateralisation of Connexin-43 were identified in rTOF RVs. A remodelling of extracellular matrix-related gene expression and an increase in collagen content that correlated with prolonged RV activation time were also found in these animals. RV action potential duration (APD) was prolonged in the epicardial anterior region at early and late repolarisation level, thus contributing to a greater APD heterogeneity and to altered transmural and anteroposterior APD gradients in rTOF RVs. APD remodelling involved changes in Kv4.3 and MiRP1 expression. Spontaneous arrhythmias were more frequent in rTOF wedges and more complex in the anterior than in the posterior RV.

Conclusion Significant remodelling of RV conduction and repolarisation properties was found in pigs with rTOF. This remodelling generates a proarrhythmic substrate likely to facilitate re-entries and to contribute to sudden cardiac death in patients with rTOF.

\section{INTRODUCTION}

Tetralogy of Fallot (TOF) is the most frequent cyanotic congenital heart defect with a prevalence of 3.5 per 10000 births. $^{1}$ Over the last decades, improvements in the timing and approach of surgical correction have considerably improved survival to adulthood. However, the adult survivor population is at risk of developing sustained ventricular arrhythmias and sudden cardiac death (SCD) late after surgery, with a respective incidence of $11.9 \%$ and $8.3 \%, 35$ years after repair. ${ }^{2}$

The role of scar regions due to right ventriculotomy and ventricular septal defect repair in supporting macroreentry circuits and monomorphic ventricular tachycardia is well established. ${ }^{3}$ Procedural success of radiofrequency ablation remains challenging $^{4}$ but recent studies targeting well-defined isthmuses appeared promising. ${ }^{3}$

In the context of SCD, there is evidence for the implication of right ventricular (RV) volume and pressure overload in sustained ventricular arrhythmias in patients with repaired Fallot (rTOF). ${ }^{5}$ QRS interval prolongation and dispersion are welldocumented predictors of ventricular arrhythmias among corrected adult patients and have been linked to RV dilatation. ${ }^{6}$ Moreover, it has been suggested that RV unloading secondary to pulmonary valve replacement may prevent worsening of these markers and when associated with intraoperative cryoablation, reduce ventricular arrhythmia incidence. ${ }^{7}$ Global heterogeneity of repolarisation, measured as QTc or JTc dispersion, was also found increased in rTOF patients and was considered as a useful marker to refine arrhythmic risk stratification. $^{58}$

In heart failure, a profound remodelling contributes to re-entrant arrhythmias and involves an extracellular matrix (ECM) proliferation and Connexin-43 downregulation both slowing electrical propagation and changes in $\mathrm{K}+$ channels expression which regulate cardiac repolarisation. ${ }^{9}$ We hypothesised that in patients with rTOF, in addition to scar-based re-entrant circuits, the overloaded RV also undergoes a structural and electrophysiological remodelling that favours re-entries and SCD. We aimed at identifying the underlying mechanisms in a porcine model reproducing TOF postoperative features. ${ }^{10} \mathrm{~A}$ better understanding of the electrophysiological and structural substrates late after TOF repair appears essential to identify new risk markers and develop specific antiarrhythmic therapeutic approaches.

\section{METHODS}

An expanded Methods section is available in the online supplementary material.

All experiments were in line with the European Union Council Directive 2010/63/EU for the protection of animals used for scientific purposes and with local ethical committee approval.

The animal model of rTOF $(\mathrm{N}=7)$ was produced as previously described. ${ }^{10}$ Briefly, piglets $(<12 \mathrm{~kg})$ were premedicated with ketamine $(10 \mathrm{mg} / \mathrm{kg}$, 
intramuscular, Vibrac) and acepromazine $(0.1 \mathrm{mg} / \mathrm{kg}$, Vetoquinol). Anaesthesia was induced with sodium pentobarbital $(5 \mathrm{mg} / \mathrm{kg}$, intravenous, Ceva) and maintained with isoflurane $\left(2 \%\right.$ in $100 \% \quad \mathrm{O}_{2}$, Vibrac). Following left lateral thoracotomy, two pulmonary valve leaflets were excised, a polytetrafluoroethylene (PTFE) patch was sewn across the pulmonary annulus and the pulmonary artery was banded. For Sham-operated animals, only the lateral thoracotomy was performed (Sham $\mathrm{N}=5$ ).

Cardiac function and remodelling was assessed $23 \pm 1$ weeks postsurgery. A conventional cardiac magnetic resonance (CMR) examination was carried out on anesthetised animals using a Siemens Magnetom Avanto 1.5 T MRI scanner. Euthanasia was performed using sodium pentobarbital (intravenous, $10 \mathrm{~mL}$ from $200 \mathrm{mg} / \mathrm{mL}$ stock) and the heart rapidly excised. Tissue samples were snap-frozen into liquid nitrogen for histological and molecular biology studies and kept at $-80^{\circ} \mathrm{C}$ until use.

The RV was dissected, perfused $(20 \mathrm{~mL} / \mathrm{min})$ and the electrical activity optically mapped using a voltage-sensitive dye (di4-ANEPPS, $10 \mu \mathrm{M})$.

Collagen content was assessed histologically in Masson's Trichrome stained sections (Sham, rTOF N=4) and RV cryosections were probed for Connexin-43 by immunofluorescence. Proteins were quantified by western blotting, mRNA levels were determined by quantitative RT-PCR and normalised to HPRT1 and GUSB expression. A microarray profiling of RV endocardium gene expression was performed on an Agilent G2565C system.

Data are expressed as means \pm SD. Statistical differences between groups were tested using one-way analysis of variances followed by Holm-Sidak multiple comparison testing or its nonparametric equivalent and linear regression as appropriate. Statistically significant difference was assumed for $\mathrm{p}<0.05$.

\section{RESULTS}

\section{Animal model}

A significant increase in heart weight and RV weight normalised to body surface area (BSA) were found in rTOF pigs, highlighting the presence of a RV hypertrophy in these animals (table 1). Pulmonary artery flow analysis at the valve level revealed a diastolic retrograde flow in all rTOF pigs which was absent in Sham pigs. The degree of regurgitation was significantly higher in rTOF animals (table 1). RV hypertrophy, dilatation and leftward septal bulging could be identified on in vivo CMR images of rTOF pigs (see online supplementary figure S1). In these animals, RV dilatation was reflected by an increase in both end-systolic (RV-ESVi) and end-diastolic (RV-EDVi) RV volumes normalised to BSA (table 1). RV stroke volume normalised to BSA remained unchanged but RV ejection fraction was significantly reduced in rTOF pigs versus Shams indicating RV dysfunction. A severity grading showed rTOF pigs were at a moderate stage of RV dysfunction (see online supplementary figure S2). Moreover, left ventricular ejection fraction was not different between rTOF and Sham pigs.

\section{Action potential duration heterogeneity in rTOF right ventricles}

A greater action potential duration (APD) heterogeneity was identified on epicardial 80\% repolarisation APD (APD80) maps from rTOF preparations (figure 1A). Quantitative analysis of APD80 distribution across the entire RV revealed an increased dispersion of APD80 in the rTOF epicardium, but not the endocardium, compared with Sham (figure 1B). However, dispersion
Table 1 Animal weights and CMR characterisation

$\begin{array}{lll}\text { Sham, } & \text { rTOF, } & \text { p Value } \\ \mathrm{N}=4 & \mathrm{~N}=6 & \end{array}$

\begin{tabular}{|c|c|c|c|}
\hline \multicolumn{4}{|l|}{ Animal weights } \\
\hline Body weight (kg) & $48 \pm 6$ & $61 \pm 11$ & 0.07 \\
\hline BSA $\left(m^{2}\right)$ & $0.93 \pm 0.07$ & $1.05 \pm 0.05$ & 0.02 \\
\hline Heart weight/BSA $\left(\mathrm{g} / \mathrm{m}^{2}\right)$ & $202 \pm 14$ & $283 \pm 21$ & 0.01 \\
\hline $\mathrm{RV}$ weight/BSA $\left(\mathrm{g} / \mathrm{m}^{2}\right)$ & $78 \pm 4$ & $133 \pm 16$ & 0.02 \\
\hline \multicolumn{4}{|l|}{ CMR characterisation } \\
\hline Heart rate (beats/min) & $117 \pm 15$ & $98 \pm 12$ & 0.09 \\
\hline PRF (\%) & 0 & $9 \pm 7$ & 0.03 \\
\hline RV-ESVi $\left(m L / m^{2}\right)$ & $37 \pm 6$ & $122 \pm 19$ & $<0.001$ \\
\hline RV-EDVi $\left(\mathrm{mL} / \mathrm{m}^{2}\right)$ & $75 \pm 5$ & $179 \pm 42$ & 0.002 \\
\hline RV-SVi $\left(\mathrm{mL} / \mathrm{m}^{2}\right)$ & $39 \pm 10$ & $57 \pm 24$ & 0.14 \\
\hline $\operatorname{RVEF}(\%)$ & $51 \pm 11$ & $31 \pm 6$ & 0.03 \\
\hline $\operatorname{LVEF}(\%)$ & $54 \pm 7$ & $61 \pm 9$ & 0.26 \\
\hline
\end{tabular}

of repolarisation time (RT) was found increased on both the epicardium and endocardium of rTOF RVs (figure 1C). Mean APD80 across the whole epicardial and endocardial surface was the same between both groups (figure 1D).

This increased heterogeneity in the RV epicardium was associated with a differential remodelling in the anterior and posterior RV. At $1 \mathrm{~Hz}$, rTOF APD20 and APD80 were prolonged in the anterior RV epicardium resulting in a significant difference with Sham anterior (figure 2A) and with rTOF posterior epicardial APD80 (see online supplementary figure S3). In contrast to the epicardium, the anterior APD20 was shorter in rTOF than Sham RV endocardium with no significant difference at APD80 level (figure 2B). As a consequence of the epicardial APD80 prolongation, the transmural APD80 gradient (APD80 was reversed in the anterior rTOF RV and significantly different from Sham anterior and from rTOF posterior gradients (figure 2C). In addition, the anteroposterior APD80 gradient (APD80 ${ }_{\text {POST-ANT) of }}$ rTOF RVs was also reversed and significantly different from Sham on the epicardium but not the endocardium (figure 2D).

Interestingly, KCND3 (Kv4.3) mRNA expression was reduced in the epicardium but increased in the endocardium of rTOF RVs compared with Sham (figure 3A, B). This observation was further confirmed at the protein level (figure 3C, D). Moreover, KCNE2 (MiRP1) mRNA expression was significantly greater in rTOF epicardium and endocardium than Sham (figure 3A, B). The mRNA expression levels for L-type $\mathrm{Ca}^{2+}$ and other $\mathrm{K}^{+}$ channels known to contribute to ventricular APD were not significantly affected (see online supplementary figure S4).

\section{RV activation and conduction velocity}

Epicardial and endocardial RV activation maps revealed a disorganised electrical activation of rTOF preparations with multiple regions of conduction slowing located in both anterior and posterior regions (figure 4A). Total activation time (AT) of the RV was prolonged in rTOF epicardium and endocardium compared with Sham (figure 4B). Moreover, a significant decrease in longitudinal and transverse conduction velocities was found in rTOF RV epicardium (figure 4C) and endocardium (figure 4D). 
Figure 1 RV epicardial and endocardial dispersion of repolarisation. (A) Representative epicardial APD80 maps from a Sham and a rTOF RV. (B) APD80 dispersion was increased in the epicardium but not the endocardium of rTOF RVs compared with Sham. (C) RT dispersion was larger in rTOF epicardium and endocardium than in Sham. (D) Mean APD80 measured over the whole epicardial and endocardial surface were similar in Sham and rTOF RVs. Data are means $\pm S D$. * $p<0.05$, ${ }^{* *} p<0.01$, Sham $N=4$, rTOF $N=5-6$. $A P D$, action potential duration; RT, repolarisation time; rTOF, repaired tetralogy of Fallot; RV, right ventricle.
A

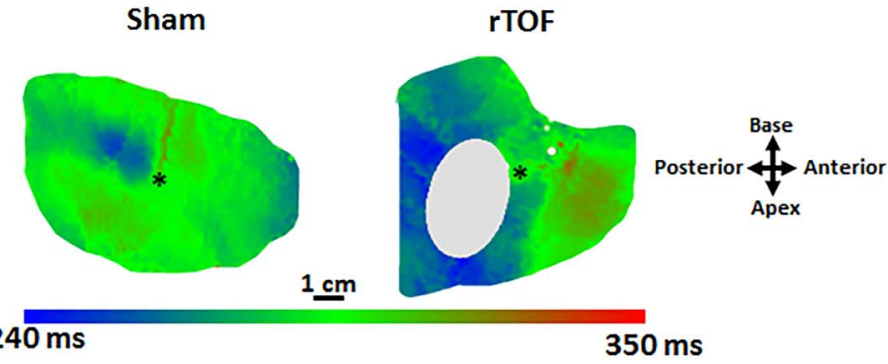

B

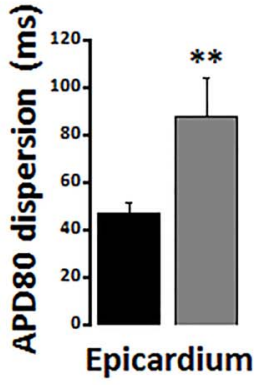

C

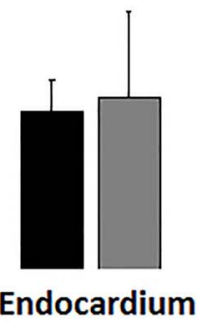

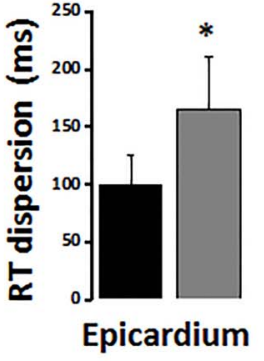

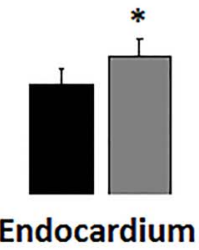

Connexin-43 expression was lower in the rTOF RV epicardium than in Sham (figure 5A) but remained unchanged in the endocardium (figure 5B). However, immunofluorescence staining on RV endocardial tissue sections from rTOF pigs revealed a Connexin-43 redistribution to the lateral sarcolemma compared with Sham sections (figure 5C).

Masson's Trichrome staining showed some degree of muscle fibre disarray and a significant increase in interstitial collagen deposition (figure 6A, B) in RV apical sections from rTOF hearts compared with Sham. A positive linear correlation was found between the endocardial AT and both the RV-EDVi (figure 6C) and the collagen content (figure 6D). Additionally, our transcriptomic approach identified significant variations in the expression of 22 genes coding for proteins forming or involved in the regulation of the ECM in the rTOF endocardium compared with Sham (see online supplementary table S1 and online supplementary results). Interestingly, the regulation of 18 of these 22 genes is commonly associated with ECM proliferation.

\section{Arrhythmias}

Spontaneous arrhythmias, often developing secondary to an increase in pacing frequency during our restitution protocol were more frequently observed in rTOF RVs than in Sham (see online supplementary figure S5 and online supplementary results).

\section{DISCUSSION}

We found a significant RV remodelling in a porcine model of rTOF with heterogeneous repolarisation and slow and discontinuous conduction which are well-known to predispose to re-entries. This remodelling was associated with the occurrence of spontaneous arrhythmias ex vivo and may thus contribute to the higher risk for SCD in patients with surgically corrected TOF.

\section{Slow and discontinuous RV activation in repaired TOF}

Slowed conduction is a prerequisite for re-entry. In patients with rTOF, QRS duration exceeding $180 \mathrm{~ms}$ is considered a predictor of severe ventricular arrhythmias which was linked to RV dilatation. ${ }^{6}{ }^{11}$ We have previously identified this QRS feature in our animal model. ${ }^{10}{ }^{12}$ In the present study, we found significant conduction disturbances associated with RV-EDVi. Our results suggest that longer RV AT was related to the presence of multiple regions of conduction slowing throughout the RV. Interestingly, this observation is reminiscent of the fractionated electrograms observed all over the RV in an endocardial mapping study in TOF-corrected patients ${ }^{13}$ and may be due to diffuse fibrosis throughout the RV as seen in advanced-stage dilated cardiomyopathy. ${ }^{14}$ Moreover, the pressure overload found in patients with TOF and in our animal model, ${ }^{12}$ is also known to trigger myocardial fibrosis. ${ }^{15}$ In patients with rTOF, CMR-based imaging techniques have allowed the localisation of 
Figure 2 Regional action potential duration and gradients in the RV. (A) Epicardial APD20 and APD80 were prolonged in the anterior RV of rTOF pigs compared with Sham (see representative traces). (B) Endocardial APD20 but not APD80 was shorter in the anterior rTOF RV than in Sham (see representative traces). (C) RV transmural APD80 gradient

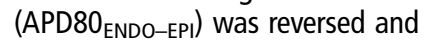
significantly different from Sham in the anterior region while remaining unchanged in rTOF posterior region. (D) RV anteroposterior APD80 gradient

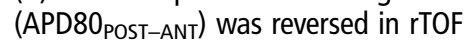
epicardium and significantly different from Sham epicardial and rTOF endocardial gradients. Data are means \pm SD. ${ }^{*} p<0.05$, * $p<0.01$, Sham $N=4$, rTOF $N=5-6$. APD, action potential duration; rTOF, repaired tetralogy of Fallot; RV, right ventricle.
A
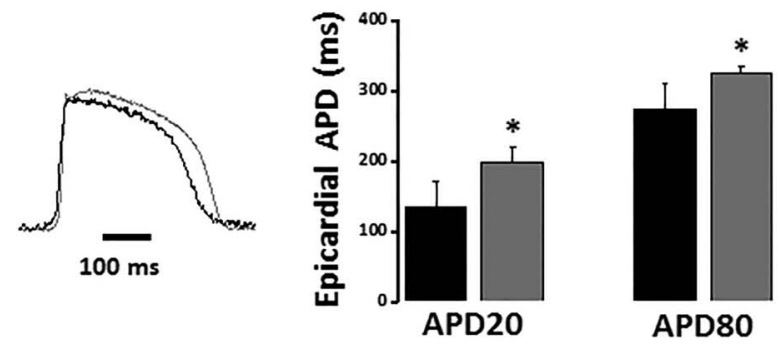

B
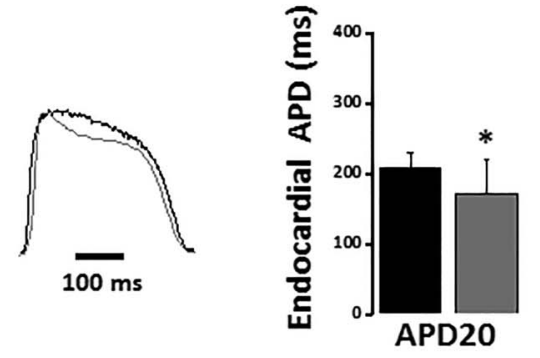

fibrosis within and beyond the scar region ${ }^{16}$ and higher degrees of fibrosis were associated with the occurrence of arrhythmias. ${ }^{17}$ Our transcriptomic approach unveiled a remodelling in the expression of genes coding for ECM-related proteins which is expected to favour ECM proliferation and contribute to conduction slowing. This remodelling appeared specific to combined pressure and volume RV overload and we could identify potential new markers of ECM remodelling (eg, serpin-family proteins) in this context (see online supplementary results). Interestingly, the expression of several proteins known to be regulated by mechanical stress (eg, Brain Natriuretic peptide) was also found altered in rTOF pigs, further highlighting the role of RV dilatation in ECM regulation. ECM regulation in the $\mathrm{RV}$ of rTOF pigs contributes to a global gene expression remodelling described in a recent study. ${ }^{18}$ While fibrosis is likely to underlie slower transverse conduction velocity in rTOF RVs, slower longitudinal conduction velocities are likely related to the decrease in Connexin-43 expression and to its lateralisation as observed in human failing hearts. ${ }^{19} \mathrm{~A}$ similar decrease in Connexin-43 expression was found in RV biopsies from patients with TOF. $^{20}$

\section{Heterogeneous RV repolarisation in repaired TOF}

Available information regarding RV repolarisation in rTOF are limited and controversial. ECG studies have shown a prolongation, ${ }^{21}$ a shortening ${ }^{5}$ or no change ${ }^{11}$ in QTc or JTc duration in these patients. These variations may be due to differences in surgical approach and patient's age at surgery and follow-up. However, similar discrepancies were found in animal models of rTOF. An APD prolongation was found in isolated RV myocytes from a similar rTOF pig model ${ }^{22}$ whereas a recent study in a dog model noted the absence of APD change in the endocardium. ${ }^{23}$ Our results suggest these discrepancies are likely related to regional APD variations in rTOF RVs. The trend for shorter APD observed in the posterior RV of our animal model may be due to chronic RV volume overload as seen in an acute study ${ }^{24}$ while pressure-overload may lead to APD prolongation in the anterior region as described in an animal model of RV hypertension. ${ }^{25}$

The APD prolongation in the anterior RV epicardium of rTOF pigs involved changes in $\mathrm{K}^{+}$channel expression. We found a trend for a reduction in $\mathrm{KCNH} 2$ (Kv11.1) mRNA expression in this region (see online supplementary figure S4A) and a significant upregulation of KCNE2 (MiRP1) a repressor of $\operatorname{IKr}(\mathrm{Kv} 11.1) .{ }^{26}$ An opposite transmural remodelling of the APD20 was found in rTOF anterior RVs which was mirrored by Kv4.3 mRNA and protein expression suggesting a transcriptional regulation of this protein. This remodelling resembles that described in human left ventricular failure where the transient outward $\mathrm{Ca}^{2+}$-insensitive current $\left(\mathrm{I}_{\mathrm{to} 1}\right)$, which is mainly carried by $\mathrm{Kv} 4.3$, was shown to be reduced in epicardial myocytes while unchanged in the endocardium. ${ }^{27}$ In addition to 
Figure 3 Potassium channel expression in the anterior RV. (A) Epicardial mRNA expression level for KCND3 was reduced in rTOF RVs while increased for KCNE2 compared with Sham. (B) Endocardial expression levels for KCND3 and KCNE2 were both increased in rTOF RVs. Kv4.3 protein expression was decreased in the epicardium (C) but increased in the endocardium (D) of rTOF RVs compared with Sham. ${ }^{* * *} p<0.001$, ${ }^{*} \mathrm{p}<0.01,{ }^{*} \mathrm{p}<0.05$, Sham $\mathrm{N}=3-4$, rTOF $\mathrm{N}=3-7$. rTOF, repaired tetralogy of Fallot; RV, right ventricle.

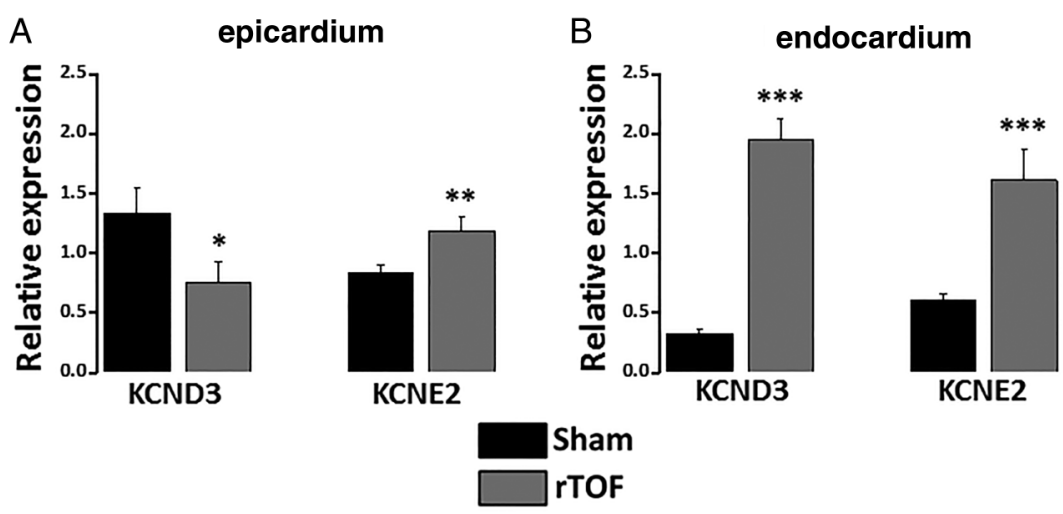

C epicardium
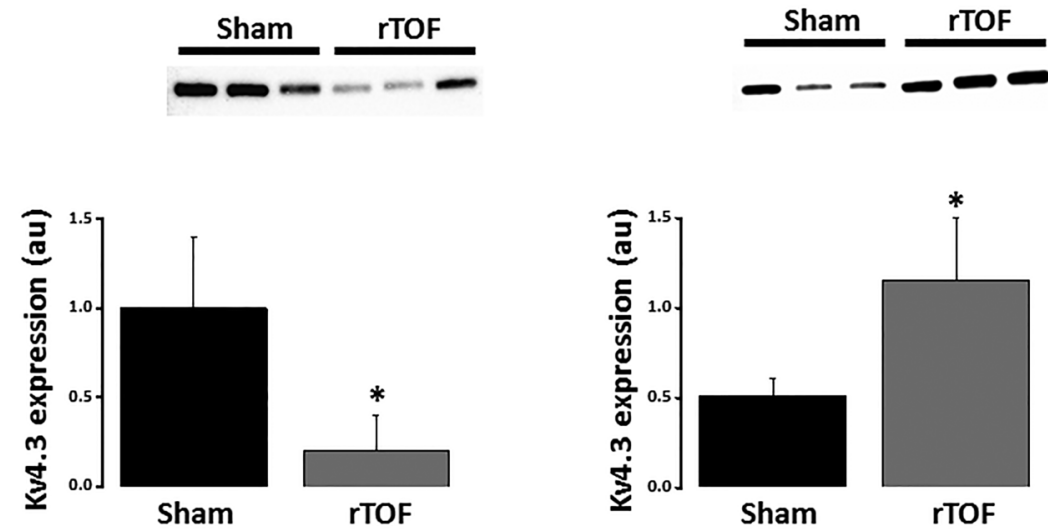

modulating APD20, regulation of this current can also alter late APD repolarisation.

Dispersion of repolarisation has commonly been described on ECGs of patients with rTOF $^{58}$ and is well-known to promote re-entries. ${ }^{9}$ In rTOF pigs, we show an increase in both epicardial and endocardial RT dispersion which was related to longer ATs and to the larger epicardial APD dispersion. Dispersion of repolarisation was further enhanced by an alteration of the transmural APD gradient in the anterior but not the posterior RV of rTOF preparations. A reduced transmural APD gradient has been described in human left-sided heart failure and was related to a larger increase in APD in the epicardium than in the endocardium $^{28}$ as observed in the anterior RV of rTOF animals. Another consequence of this regional APD remodelling is a decrease and reversal of the anteroposterior APD gradient in the epicardium but not in the endocardium. Regionalisation of transmural and anteroposterior APD gradient alterations enhances APD dispersion in rTOF RVs and increases the risk of re-entry.

\section{Arrhythmias}

The number of arrhythmia per rTOF preparation correlated with transverse endocardial conduction velocity. This finding further highlights the link between prolonged RV activation and arrhythmia reported in patients. ${ }^{2}{ }^{6}$ Moreover, the correlation between RV AT and RV-EDVi indirectly links RV dilatation to an increased arrhythmic risk. Interestingly, it has been shown in pigs that sustained arrhythmias could only be induced in 23\% of animals with a right ventricular outflow tract (RVOT) scar against $83 \%$ of animals with pulmonary insufficiency and
RVOT scar. ${ }^{29}$ This illustrates the proarrhythmic synergy of RV overload and surgical scaring.

\section{Clinical implications}

Our results suggest that re-entries in patients with surgically corrected TOF are not solely related to the surgical scars and involve a global RV remodelling. Characterisation of this electrophysiological and structural remodelling could help identifying patients at risk of complex arrhythmias and SCD and may suggest therapeutic interventions such as pulmonary valve replacement, radiofrequency ablation, or cardiac resynchronisation. Moreover, antiarrhythmic therapy aimed at this remodelling may prevent SCD or improve radiofrequency ablation success.

According to the results from the present study and others, ${ }^{16} 17$ therapeutically targeting fibrosis by interfering with the renin-angiotensin system in patients with rTOF appears a promising approach. Furthermore, since fibrosis is likely triggered by chronic volume overload and stretch, ${ }^{30}$ early surgical interventions aiming at correcting pulmonary regurgitation prior to significant RV remodelling could be another potential therapeutic approach.

\section{Limitations}

Our animal model aimed at reproducing postoperative Fallot haemodynamics and electrophysiology in healthy pigs in the absence of TOF. However, factors independent of surgery (eg, genetic substrate, hypoxaemia) may play a role in the ventricular remodelling and arrhythmia generation. 
Figure 4 RV activation in Sham and rTOF preparations. (A) Representative activation maps from a Sham and a rTOF RV with 5 ms-spaced isochrones. Multiple areas of slowed conduction were observed (yellow arrows) throughout rTOF RVs. (B) Epicardial and endocardial activation times were longer in rTOF than in Sham RVs. Epicardial (C) and endocardial (D) CVs were reduced in the longitudinal and transverse directions of propagation in rTOF compared with Sham

preparations. Data are means \pm SD.

${ }^{* *} p<0.01,{ }^{*} p<0.05$, Sham $N=4$, rTOF $\mathrm{N}=5-6$. ENDO, endocardial; EPI, epicardial; CV, conduction velocity; rTOF, repaired tetralogy of Fallot; RV, right ventricle.

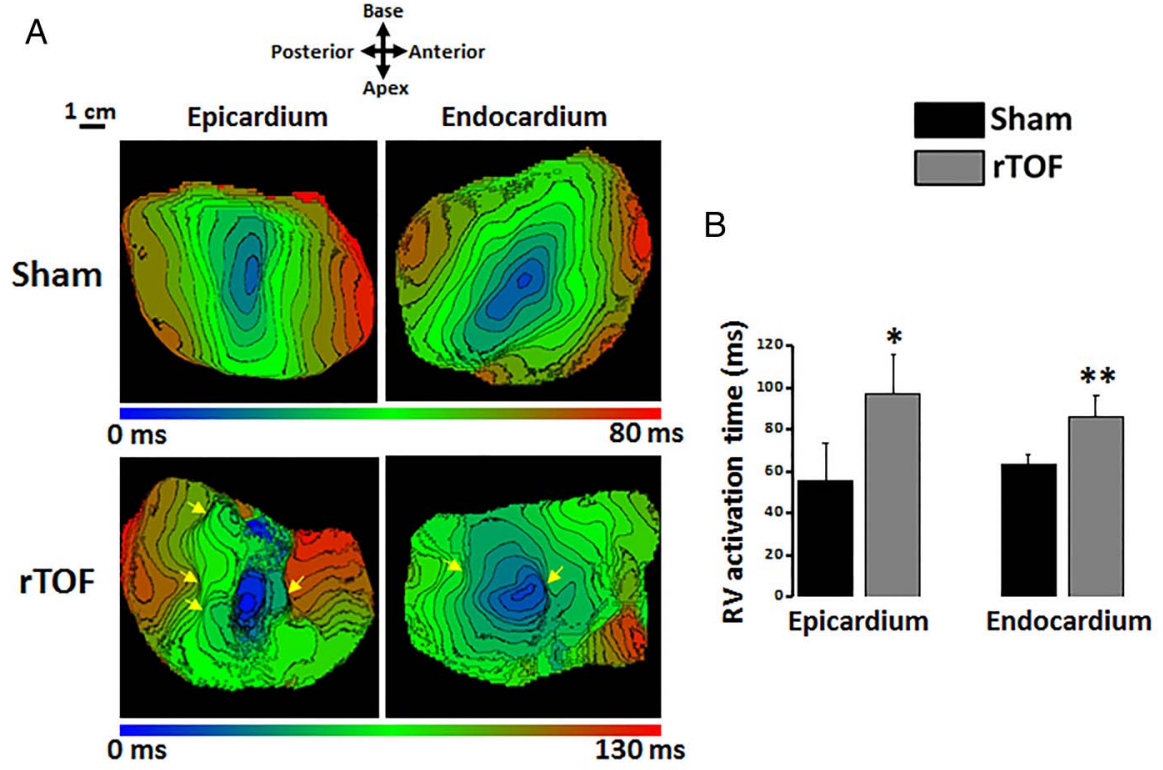

C
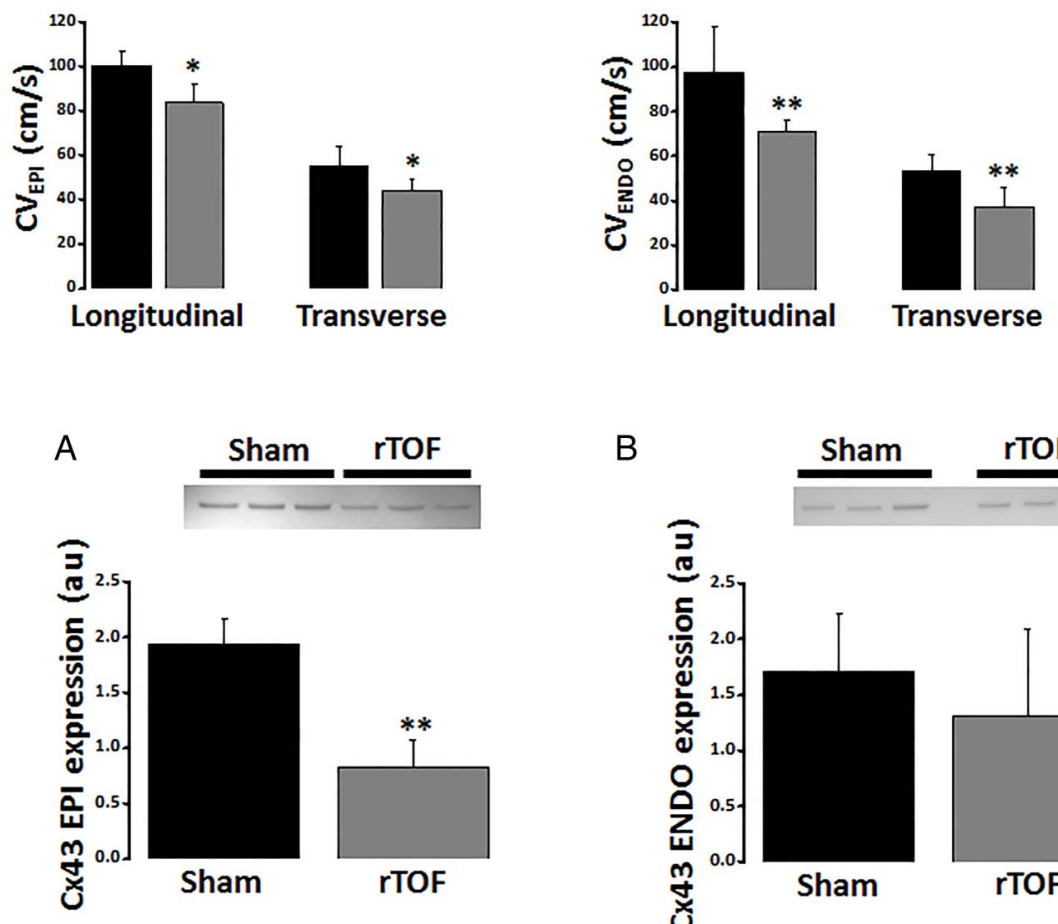

B

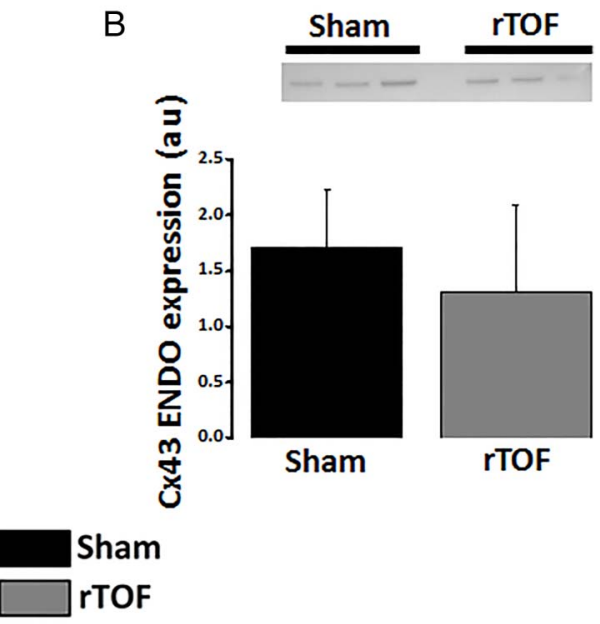

epicardial; Cx43, Connexin-43; rTOF repaired tetralogy of Fallot; RV, right ventricle.

Figure $5 \mathrm{Cx} 43$ expression and localisation. (A) $\mathrm{Cx} 43$ protein expression was decreased in the epicardium but not the endocardium (B) of rTOF RVs compared with Sham. (C) Immunolabelling revealed the presence of $\mathrm{Cx} 43$ at the lateral membrane (grey arrows) in rTOF endocardial sections as opposed to the intercalated disc distribution (white arrows) seen in Sham sections. Data are means $\pm S D$. ${ }^{* *} p<0.01$, Sham $N=3$, rTOF N=3. ENDO, endocardial; $\mathrm{EPI}$

C

Sham

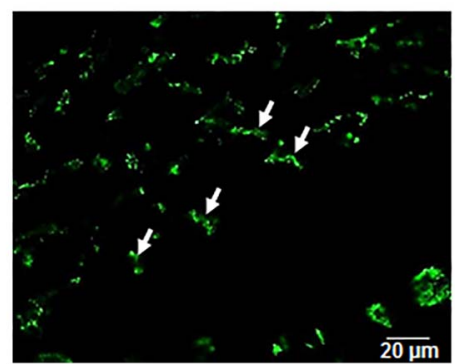


Figure 6 Collagen content in Sham and rTOF right ventricles. (A) Histological sections stained with Masson's Trichrome showing a greater collagen staining (blue) and a disorganised myocardial structure in the rTOF RV. (B) Collagen content was increased in rTOF RVs compared with Sham. RV endocardial activation time correlated linearly with RV-EDVi (C) and collagen content (D). Data are means \pm SD. * $p<0.05$, Sham $N=4$, rTOF $\mathrm{N}=4-6$. ENDO, endocardial; $\mathrm{EDVi}$, end-diastolic volume indexed to body surface area; rTOF, repaired tetralogy of Fallot; RV, right ventricle.
A

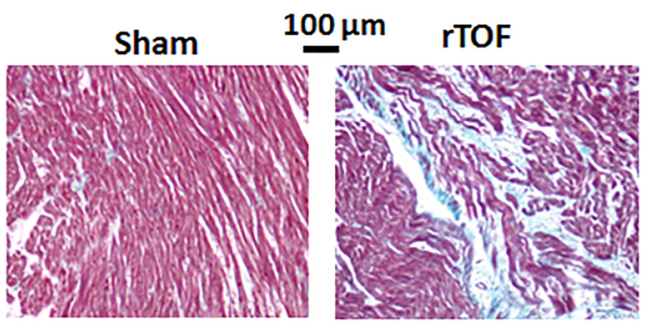

C

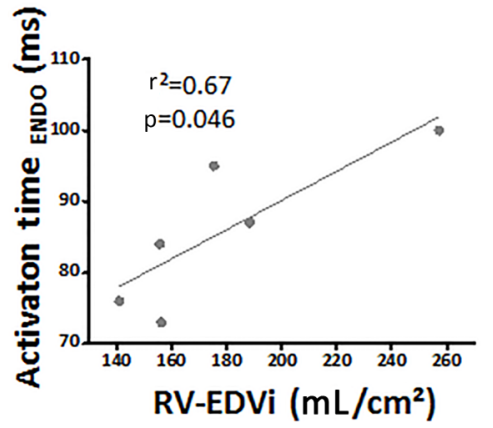

B

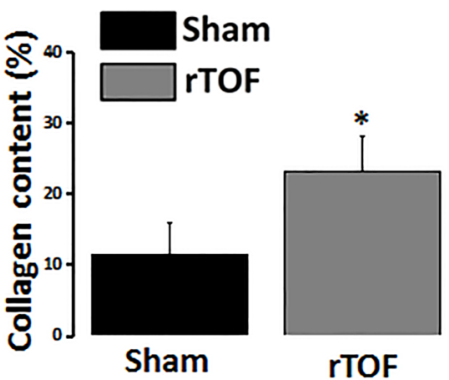

D

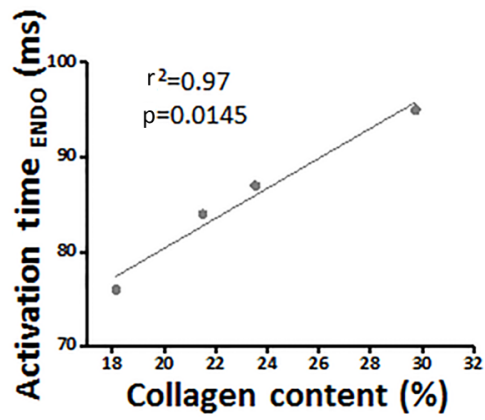

\section{Key messages}

\section{What is already known on this subject?}

Patients with repaired tetralogy of Fallot (TOF) are exposed to life-threatening arrhythmias late after surgery. Surgical scars have been shown to support macroreentrant circuits and monomorphic ventricular tachycardia. Moreover, ECG indexes also suggest the presence of a ventricular remodelling linked to right ventricular (RV) overload in this population.

\section{What might this study add?}

Here we characterised the electrophysiological and structural remodelling of the RV in a preclinical pig model of TOF repair. The slower, discontinuous conduction and heterogeneous repolarisation found across the whole RV generate a substrate for re-entries. Mechanisms associated with this remodelling involve a proliferation of the extracellular matrix and changes in ion channel expression. Thus, our results suggest a global remodelling of RV structure and electrophysiology contributes to arrhythmias in repaired TOF.

\section{How might this impact on clinical practice?}

Characterisation of this substrate and potential new risk markers found in this study could help identifying patients at risk of sudden cardiac death (SCD) and may suggest therapeutic interventions such as pulmonary valve replacement, radiofrequency ablation, or cardiac resynchronisation. Moreover, antiarrhythmic therapy aimed at this remodelling may prevent SCD or improve radiofrequency ablation success.

We did not apply specific pacing protocols to investigate the vulnerability to ventricular arrhythmias due to experimental time constraints on the ex vivo tissue preparations. However, the occurrence of spontaneous arrhythmias arising during our pacing protocol indicates a strong arrhythmogenic substrate in the rTOF animals.

\section{CONCLUSION}

RV conduction and repolarisation properties are altered in a porcine model reproducing TOF repair lesions, leading to a substrate for re-entries. Mechanisms involved an ECM proliferation, a Connexin-43 downregulation and lateralisation which slowed electrical propagation; and a regionalised remodelling in $\mathrm{Kv} 4.3$ and MiRP1 expression which increased APD dispersion. This remodelling is likely to contribute to arrhythmias and SCD in corrected patients with TOF and represent a potential therapeutic target.

\section{Author affiliations}

${ }^{1}$ IHU LIRYC, L'Institut de Rythmologie et Modélisation Cardiaque, Fondation Bordeaux Université, Bordeaux, France

${ }^{2}$ Inserm U1045, Centre de Recherche Cardio-Thoracique de Bordeaux, Université de Bordeaux, Bordeaux, France

${ }^{3} \mathrm{CHU}$ de Bordeaux, Hôpital Cardiologique du Haut-Lévêque, Pessac, France

${ }^{4}$ Max Delbrück Center for Molecular Medicine, Mathematical Cell Physiology, Berlin, Germany

${ }^{5}$ Institute of Biomedical Engineering, Department of Engineering Science, University of Oxford, Oxford, UK

${ }^{6}$ Plateforme Technologique d'Innovation Biomédicale, Université de Bordeaux, Pessac, France

${ }^{7}$ Inserm U1211, Maladies Rares: Génétique et Métabolisme, Université de Bordeaux, Bordeaux, France

Contributors $D B, V D, F R$, SHG, SC, MC, DE, DV, BQ performed the experiments; $D B, V D, S H G, S C, O B$ analysed the data; $D B, V D, F R, B Q, H C, M H, C R, P B, J-B T$, $\mathrm{OB}$ designed the experimental protocol; $\mathrm{DB}, \mathrm{VD}$ and $\mathrm{OB}$ wrote the manuscript.

Funding This work was funded by the Agence Nationale de la Recherche through the grant ANR-10-IAHU04-LIRYC, the ERC Advanced grant SYMPHONY (no 322886) and the EU FP7 IRSES programme CORDIS3D grant (IRSES-GA-2013-317767). DB was funded by a Fondation Recherche Médicale fellowship and SHG was funded by a European Union Marie Curie fellowship.

Competing interests None declared.

Provenance and peer review Not commissioned; externally peer reviewed.

Open Access This is an Open Access article distributed in accordance with the Creative Commons Attribution Non Commercial (CC BY-NC 4.0) license, which permits others to distribute, remix, adapt, build upon this work non-commercially, and license their derivative works on different terms, provided the original work is properly cited and the use is non-commercial. See: http://creativecommons.org/licenses/by-nc/4.0/

\section{REFERENCES}

1 Egbe A, Uppu S, Lee $S$, et al. Changing prevalence of severe congenital heart disease: a population-based study. Pediatr Cardiol 2014;35:1232-8. 
2 Gatzoulis MA, Balaji S, Webber SA, et al. Risk factors for arrhythmia and sudden cardiac death late after repair of tetralogy of Fallot: a multicentre study. Lancet 2000;356:975-81.

3 Zeppenfeld K, Schalij MJ, Bartelings MM, et al. Catheter ablation of ventricular tachycardia after repair of congenital heart disease: electroanatomic identification of the critical right ventricular isthmus. Circulation 2007;116:2241-52.

4 Morwood JG, Triedman JK, Berul Cl, et al. Radiofrequency catheter ablation of ventricular tachycardia in children and young adults with congenital heart disease. Heart Rhythm 2004;1:301-8.

5 Helbing WA, Roest AA, Niezen RA, et al. ECG predictors of ventricular arrhythmias and biventricular size and wall mass in tetralogy of Fallot with pulmonary regurgitation. Heart 2002;88:515-19.

6 Gatzoulis MA, Till JA, Somerville J, et al. Mechanoelectrical interaction in tetralogy of Fallot. QRS prolongation relates to right ventricular size and predicts malignant ventricular arrhythmias and sudden death. Circulation 1995;92:231-7.

7 Therrien J, Siu SC, Harris L, et al. Impact of pulmonary valve replacement on arrhythmia propensity late after repair of tetralogy of Fallot. Circulation 2001;103:2489-94.

8 Gatzoulis MA, Till JA, Redington AN. Depolarization-repolarization inhomogeneity after repair of tetralogy of Fallot. The substrate for malignant ventricular tachycardia? Circulation 1997;95:401-4.

9 Coronel R, Wilders R, Verkerk AO, et al. Electrophysiological changes in heart failure and their implications for arrhythmogenesis. Biochim Biophys Acta 2013; 1832:2432-41

10 Thambo JB, Roubertie F, De Guillebon $M$, et al. Validation of an animal model of right ventricular dysfunction and right bundle branch block to create close physiology to postoperative tetralogy of Fallot. Int J Cardiol 2012;154:38-42.

11 Berul Cl, Hill SL, Geggel RL, et al. Electrocardiographic markers of late sudden death risk in postoperative tetralogy of Fallot children. J Cardiovasc Electrophysiol 1997:8:1349-56.

12 Thambo JB, Dos Santos P, De Guillebon M, et al. Biventricular stimulation improves right and left ventricular function after tetralogy of Fallot repair: Acute animal and clinical studies. Heart Rhythm 2010;7:344-50.

13 Deanfield JE, McKenna WJ, Presbitero $P$, et al. Ventricular arrhythmia in unrepaired and repaired tetralogy of Fallot. Relation to age, timing of repair, and haemodynamic status. Br Heart J 1984;52:77-81.

14 Assomull RG, Prasad SK, Lyne J, et al. Cardiovascular magnetic resonance, fibrosis, and prognosis in dilated cardiomyopathy. J Am Coll Cardiol 2006;48:1977-85.

15 Creemers EE, Pinto YM. Molecular mechanisms that control interstitial fibrosis in the pressure-overloaded heart. Cardiovasc Res 2011;89:265-72.

16 Wald RM, Haber I, Wald R, et al. Effects of regional dysfunction and late gadolinium enhancement on global right ventricular function and exercise capacity in patients with repaired tetralogy of Fallot. Circulation 2009:119:1370-7.
17 Babu-Narayan SV, Kilner PJ, Li W, et al. Ventricular fibrosis suggested by cardiovascular magnetic resonance in adults with repaired tetralogy of fallot and its relationship to adverse markers of clinical outcome. Circulation 2006;113:405-13.

18 Charron S, Roubertie F, Benoist D, et al. Identification of Region-Specific Myocardial Gene Expression Patterns in a Chronic Swine Model of Repaired Tetralogy of Fallot. PLOS ONE 2015;10:e0134146.

19 Glukhov AV, Fedorov VV, Kalish PW, et al. Conduction remodeling in human end-stage nonischemic left ventricular cardiomyopathy. Circulation 2012;125:1835-47.

20 Kołcz J, Drukała J, Bzowska M, et al. The expression of connexin 43 in children with Tetralogy of Fallot. Cell Mol Biol Lett 2005;10:287-303.

21 Sarubbi B, Pacileo G, Ducceschi V, et al. Arrhythmogenic substrate in young patients with repaired tetralogy of Fallot: role of an abnormal ventricular repolarization. Int J Cardiol 1999:72:73-82.

22 Lambert V, Capderou A, Le Bret $E$, et al. Right ventricular failure secondary to chronic overload in congenital heart disease: an experimental model for therapeutic innovation. J Thorac Cardiovasc Surg 2010;139:1197-204, 204.e1.

23 Chiu SN, Tsai CT, Lin LY, et al. Repolarization alternans and ventricular arrhythmia in a repaired tetralogy of fallot animal model. J Am Heart Assoc 2015;4:e002173.

24 Greve G, Lab MJ, Chen R, et al. Right ventricular distension alters monophasic action potential duration during pulmonary arterial occlusion in anaesthetised lambs: evidence for arrhythmogenic right ventricular mechanoelectrical feedback. Exp Physiol 2001;86:651-7.

25 Benoist D, Stones R, Benson AP, et al. Systems approach to the study of stretch and arrhythmias in right ventricular failure induced in rats by monocrotaline. Prog Biophys Mol Biol 2014;115:162-72.

26 Abbott GW, Sesti F, Splawski I, et al. MiRP1 forms IKr potassium channels with HERG and is associated with cardiac arrhythmia. Cell 1999;97: 175-87.

27 Näbauer $M$, Beuckelmann DJ, Uberfuhr $P$, et al. Regional differences in current density and rate-dependent properties of the transient outward current in subepicardial and subendocardial myocytes of human left ventricle. Circulation 1996:93:168-77.

28 Glukhov AV, Fedorov VV, Lou Q, et al. Transmural dispersion of repolarization in failing and nonfailing human ventricle. Circ Res 2010;106:981-91.

29 Zeltser I, Gaynor JW, Petko M, et al. The roles of chronic pressure and volume overload states in induction of arrhythmias: an animal model of physiologic sequelae after repair of tetralogy of Fallot. $J$ Thorac Cardiovasc Surg 2005;130:1542-8.

30 Hutchinson KR, Stewart JA Jr, Lucchesi PA. Extracellular matrix remodeling during the progression of volume overload-induced heart failure. J Mol Cell Cardiol 2010;48:564-9. 